\title{
Two-photon phosphorescence lifetime microscopy of retinal capillary plexus oxygenation in mice
}

\author{
Ikbal Şencan \\ Tatiana V. Esipova \\ Mohammad A. Yaseen \\ Buyin $\mathrm{Fu}$ \\ David A. Boas \\ Sergei A. Vinogradov \\ Mahnaz Shahidi \\ Sava Sakadžić
}




\title{
Two-photon phosphorescence lifetime microscopy of retinal capillary plexus oxygenation in mice
}

\author{
Ikbal Şencan, ${ }^{a}$ Tatiana V. Esipova, ${ }^{b}$ Mohammad A. Yaseen, ${ }^{a}$ Buyin Fu, ${ }^{a}$ David A. Boas, ${ }^{\text {a,c }}$ \\ Sergei A. Vinogradov, ${ }^{b, \star}$ Mahnaz Shahidi, ${ }^{d, \star}$ and Sava Sakadžićc, ${ }^{, \star \star}$ \\ aMassachusetts General Hospital, Harvard Medical School, Athinuola A. Martinos Center for Biomedical Imaging, \\ Department of Radiology, Charlestown, Massachusetts, United States \\ bUniversity of Pennsylvania, Departments of Biochemistry and Biophysics and of Chemistry, Philadelphia, Pennsylvania, United States \\ ${ }^{\mathrm{C} B o s t o n}$ University, Department of Biomedical Engineering, Boston, Massachusetts, United States \\ dUniversity of Southern California, Departments of Ophthalmology and Biomedical Engineering, Los Angeles, California, United States
}

\begin{abstract}
Impaired oxygen delivery and/or consumption in the retinal tissue underlies the pathophysiology of many retinal diseases. However, the essential tools for measuring oxygen concentration in retinal capillaries and studying oxygen transport to retinal tissue are still lacking. We show that two-photon phosphorescence lifetime microscopy can be used to map absolute partial pressures of oxygen $\left(\mathrm{pO}_{2}\right)$ in the retinal capillary plexus. Measurements were performed at various retinal depths in anesthetized mice under systemic normoxic and hyperoxic conditions. We used a newly developed two-photon phosphorescent oxygen probe, based on a two-photon absorbing platinum tetraphthalimidoporphyrin, and commercially available optics without correction for optical aberrations of the eye. The transverse and axial distances within the tissue volume were calibrated using a model of the eye's optical system. We believe this is the first demonstration of in vivo depth-resolved imaging of $\mathrm{pO}_{2}$ in retinal capillaries. Application of this method has the potential to advance our understanding of oxygen delivery on the microvascular scale and help elucidate mechanisms underlying various retinal diseases. () The Authors. Published by SPIE and CLP under a Creative Commons Attribution 4.0 Unported License. Distribution or reproduction of this work in whole or in part requires full attribution of the original publication, including its DOI. [DOI: 10.1117/1.JBO.23.12.126501]
\end{abstract}

Keywords: two-photon microscopy; phosphorescence quenching; oxygen partial pressure; retinal imaging

Paper 180490R received Aug. 10, 2018; accepted for publication Nov. 7, 2018; published online Dec. 4, 2018.

\section{Introduction}

The retina has complex microvascular architecture and requires ample oxygen delivery to maintain its high metabolic activity. ${ }^{1}$ Insufficient blood supply to the retina can cause impaired vision and blindness ${ }^{2,3}$ due to common retinal diseases, such as diabetic retinopathy. According to the National Eye Institute, in the United States alone 7.7 million people with diabetic retinopathy were reported in 2010, and this number is expected to double by $2050 .{ }^{4}$ However, despite the urgent need to prevent vision loss, the knowledge of how disease development and progression is related to impaired oxygen delivery and consumption is still limited. ${ }^{5}$ This knowledge gap persists in part due to the lack of techniques for measuring oxygen concentration in the retina with high spatiotemporal resolution. Previous studies have shown that retinal $\mathrm{pO}_{2}$ in rodents varies across the total depth of the retinal tissue, although less so in the inner retina. ${ }^{6-8}$ However, physiological challenges and/or diseases may differentially affect $\mathrm{pO}_{2}$ in the inner retinal capillary layers. For example, light flicker stimulation differentially affects vessel dilation and blood flux at the inner retina capillary layers in rats. ${ }^{9}$ Recent studies have also demonstrated elevation in the inner-retinal $\mathrm{pO}_{2}$ levels in diabetic rats ${ }^{10}$ as well as variations in perfusion of different inner-retinal capillary layers in humans affected by diabetic retinopathy. ${ }^{11,12}$ Therefore, there is a need for development of techniques for depth-resolved $\mathrm{pO}_{2}$ measurements in the retina, which will help in unraveling mechanisms underlying neurovascular coupling and retinal diseases.

\footnotetext{
*Address all correspondence to: Sergei A. Vinogradov, E-mail: vinograd .upenn@gmail.com; Mahnaz Shahidi, E-mail: mshahidi@usc.edu; Sava Sakadžić, E-mail: sava.sakadzic@mgh.harvard.edu
}

Various methods have been reported for in vivo assessment of retinal oxygenation in rodents. Currently, oxygen microelectrode is considered the gold standard of retinal extravascular (tissue) $\mathrm{pO}_{2}$ measurements with high depth resolution.,7 However, this technique is invasive, and measurements are limited to single point locations in the retina, where the local microenvironment is potentially being disturbed by the electrodes. Numerous techniques for assessment of retinal intravascular oxygenation are based on optical absorption spectroscopy of hemoglobin, including spectrophotometric oximetry, ${ }^{13}$ photoacoustic imaging, ${ }^{14}$ and visible light optical coherence tomography. ${ }^{15-17}$ While all these methods can be relatively easily translated to the clinic, they suffer from common limitations. First, hemoglobin-based techniques report oxygen only in the blood, but not at the sites of the actual oxygen consumption. Second, hemoglobin-based measurements are better suited for accessing oxygenation in major arteries and veins and do not provide reliable measurements within retinal microvessels and capillaries.

Another optical approach to imaging retinal intravascular oxygenation is based on oxygen-dependent quenching of phosphorescence. ${ }^{18}$ Originally, this method was used for assessment of oxygen levels in retinal and choroidal vessels by widefield time-domain phosphorescence lifetime imaging, ${ }^{19}$ and later extended to the frequency domain. ${ }^{20}$ A number of retinal oxygen imaging studies by us and other groups followed, typically using gated CCD cameras and phosphorescent probes based on $\mathrm{Pd}$ porphyrins $^{20-25}$ and, more recently, an Ir(III) complex. ${ }^{26}$ By using the laser-line illumination approach, we have also demonstrated the feasibility of 3-D imaging of both the retinal vascular $\mathrm{pO}_{2}{ }^{27,28}$ and retinal extravascular $\mathrm{pO}_{2}$ with high lateral resolution, ${ }^{29,30}$ though depth resolution was limited by scattering. 
To date, depth-resolved $\mathrm{pO}_{2}$ measurements in the retinal microvasculature with truly microscopic resolution have not been demonstrated. Such measurements are essential for assessment of oxygenation in capillaries where the earliest retinal vasculopathies occur, providing critical information about oxygen delivery to the retinal cells.

Here, we performed for the first time high-resolution absolute $\mathrm{pO}_{2}$ measurements in superficial, intermediate, and deep retinal microvascular plexus by two-photon phosphorescence lifetime microscopy (2PLM) ${ }^{31}$ using a recently developed probe based on a bright two-photon-absorbing platinum tetraphthalimidoporphyrin. ${ }^{32}$ We measured intravascular $\mathrm{pO}_{2}$ in major arteries and veins as well as in precapillary arterioles, capillaries, and postcapillary venules within the retinal microvascular plexus in the retina of healthy anesthetized mice under normoxia and hyperoxia. Noteworthy, our two-photon microscope ${ }^{33}$ was equipped with a commercially available objective lens with a long working distance, having no additional tools (e.g., adaptive optics) for correcting optical aberrations of the eye. We constructed the ray transfer matrix of the mouse eye based on optical parameters (refractive index, thickness, curvature, etc.) that were previously reported in the literature, and accordingly calibrated the lateral and axial retinal distances in our measurements. The new method has the potential to provide information that will be invaluable for understanding retinal vasculopathies occurring in diabetic retinopathy and similar diseases of the eye.

\section{Experimental Methods}

\subsection{Optical Setup}

The custom-built 2PM system (Fig. 1) ${ }^{33}$ was used for both 3-D retinal microvasculature angiography and measurements of $\mathrm{pO}_{2}$. All measurements were performed in the dark.

Two-photon excitation was performed using a tunable femtosecond laser (InSight, Spectra-Physics, 680 to $1300 \mathrm{~nm}$, $80 \mathrm{MHz}$ rep. rate). The power level and temporal gating of the excitation beam were controlled by an electro-optic modulator (EOM) (ConOptics, 350 to 160). The beam was steered in the lateral plane $(X$ and $Y$ ) by two galvanometer mirrors (Cambridge Technologies, $6215 \mathrm{HB}$ ) and focused onto a desired retina layer by an objective lens (Olympus, XLPLN10XSVMP, $10 \times, 0.6 \mathrm{NA}$, water immersion), which was separated from the mouse cornea by transparent ultrasound gel, a glass cover slip, and a thin layer of eye lubricant (see Fig. 1 inset). The working distance (WD $=8 \mathrm{~mm}$ ) of the objective lens was chosen to be significantly longer than the physical cornea-to-retina distance $(\sim 3.2 \mathrm{~mm}),{ }^{34-36}$ since the apparent optical depth of the retina position is higher due to the optical properties of the eye. The emission originating from the retinal microvasculature was collected by the objective lens, reflected by an epidichroic mirror (Semrock Inc., FF875-Di01-25x36), passed through a short-pass filter (Semrock Inc., FF01-890/SP-50), and split into two channels. One was directed to a photon-counting photomultiplier tube (PMT) (PMT, Hamamatsu, H10770PA-50) through a bandpass filter (Semrock Inc., FF01-795/150-25) and used for recording the phosphorescence of the probe. Another was passed through a band-pass filter (Semrock Inc., FF01-525/50-25) and directed into analog PMT (Hamamatsu, $\mathrm{R} 3896$ ), for recording the fluorescence of vascular markers

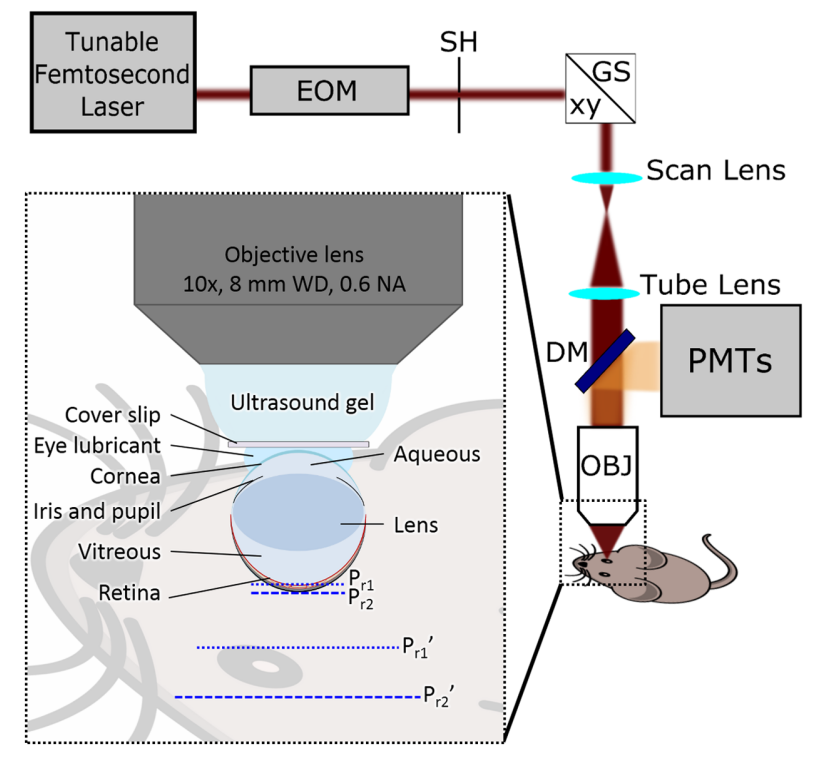

Fig. 1 Optical setup for 2PM imaging of $\mathrm{pO}_{2}$ in mouse retinal microvasculature. EOM, electro-optic modulator; $\mathrm{SH}$, shutter; GS, $x y$ galvanometer scanner; DM, dichroic mirror; OBJ, microscope objective lens; PMTs, four-channel detector unit. The inset illustrates the coupling of the long-working-distance microscope objective with the mouse eye and eye anatomical features considered, when modeling the propagation of the optical rays. $P_{r 1}$ and $P_{r 2}$ are top and bottom retina planes, respectively. $P_{r 1}^{\prime}$ and $P_{r 2}^{\prime}$ are virtual images of $P_{r 1}$ and $P_{r 2}$, respectively, created by the compound optical system of the mouse eye.

[e.g., fluorescein isothiocyanate (FITC)-dextran] in order to construct microvascular angiograms of the regions of interest.

\subsection{Animal Preparation}

All surgeries and experimental procedures were performed in accordance with the guidelines established by the MGH Institutional Animal Care and Use Committee (IACUC). We used three female C57BL/six mice (Charles River Laboratories, 3-months old, 22 to $23 \mathrm{~g}$ ). Mice were anesthetized during surgery using $1.5 \%$ to $2 \%$ isoflurane in a mixture of air and oxygen, as they underwent tracheotomy for ventilation and femoral artery cannulation for measuring the blood pressure and heart rate, injection of the contrast agents (probes), and blood gas measurements. The body temperature was maintained at $37^{\circ} \mathrm{C}$ using a heating blanket and monitored by a rectal thermometer.

Prior to imaging, the pupils were dilated using $1 \%$ tropicamide eye drops, and an eye lubricant gel (Goniovisc Hypromellose Ophthalmic solution USP $25 \mathrm{mg}, 2.5 \%$ ) was applied over the cornea. Animals were placed in a prone position; the head was slightly tilted and restrained using a metal bar previously attached to the skull with dental cement to minimize motion artifacts induced by respiration and heartbeat. A microscope coverslip was placed over the right cornea. The coverslip was in contact with the eye lubricant gel, providing a flat surface, and it was optically coupled with the objective lens by using ultrasound gel. A mixture of the phosphorescent probe [molecular weight (MW) $75 \mathrm{kDa}, 0.1 \mathrm{~mL}, 34 \mu \mathrm{M}$ ] and dextran-conjugated fluorescein (FITC, $0.1 \mathrm{~mL}, 89 \mu \mathrm{M}$ ) was administered into the blood plasma via a femoral artery cannula. The mice were kept anesthetized during imaging using $1 \%$ isoflurane in a mixture of air and oxygen. The average power limit for 
ocular safety (based on ANSI 2000) $)^{37}$ was calculated to be $61 \mathrm{~mW}$ in our imaging configuration $(10-\mu \mathrm{s}$-long excitation gate at $950 \mathrm{~nm}$ ). The average laser power after the objective during application of the excitation gate (high-repetition-rate pulse train) was 1.3 to $58 \mathrm{~mW}$. We induced hyperoxic and normoxic conditions by manipulating the $\mathrm{O}_{2}$ concentration in the inhaled mixture of $\mathrm{O}_{2}$ and air $\left(\mathrm{O}_{2}: 21 \%\right.$ during normoxia and $100 \%$ during hyperoxia) and confirmed the results through systemic arterial blood gas measurements. The animal blood pressure (BP), heart rate, body temperature, and expired $\mathrm{CO}_{2}$ were monitored continuously during the experiments. The range of $\mathrm{BP}$ and systemic $\mathrm{pCO}_{2}$ during the experiments were 67 to $90 \mathrm{mmHg}$ and 30 to $37.5 \mathrm{mmHg}$, respectively. Surgical preparations took 30 to $45 \mathrm{~min}$ and moving the animal to the microscope, positioning of the animal, injection of contrast agent, and testing blood gasses took additional 30 to $45 \mathrm{~min}$.

\subsection{Depth-Resolved Imaging of Retinal Microvasculature Using Two-Photon Fluorescence Microscopy}

In order to visualize retinal microvasculature, high-resolution volumetric angiograms were recorded by means of 2PM fluorescence imaging of the FITC-labeled blood plasma. We started our measurements by selecting a region of interest (ROI) using an air-coupled, low-resolution objective (Olympus, XLFLUOR4x/340, 4×, 0.28 NA, 30-mm WD) with a large field-of-view. Then we switched to a high-resolution waterimmersion objective (Olympus XLPLN10XSVMP, 10×, 0.6 NA, 8-mm WD) to record the angiograms. The excitation beam $(840 \mathrm{~nm})$ was raster scanned with a dwell time of $4 \mu \mathrm{s} /$ pixel. The FITC fluorescence was recorded at multiple planes through the retinal depth by translating the imaging objective along the optical $Z$ axis.

\subsection{Depth-Resolved Measurement of Absolute $\mathrm{pO}_{2}$ in Retinal Microvasculature Using Two-Photon Phosphorescence Lifetime Microscopy}

Intravascular $\mathrm{pO}_{2}$ was quantified using oxygen-dependent quenching of phosphorescence and a new probe based on a new highly two-photon absorbing $\mathrm{Pt}(\mathrm{II})$ tetraphthalimidoporphyrin. ${ }^{32}$ The probe does not cross the blood brain barrier and/or cellular membranes and can be used for both intravascular and extravascular oxygen imaging. The new probe belongs to the class of dendritically protected phosphorescent probes $^{38}$ and is characterized by high two-photon absorption cross-section ( $\sim 600 \mathrm{GM}$ at $950 \mathrm{~nm})$ and high phosphorescence quantum yield ( $\sim 0.23$ in aqueous solution at $22^{\circ} \mathrm{C}$ ). Before recording phosphorescence decays in selected locations, the excitation beam $(950 \mathrm{~nm})$ was raster scanned in the corresponding axial plane, and a two-dimensional phosphorescence intensity image (survey image) of the ROI was recorded. This survey scan helped to position the beam at the desired location(s) for collection of the time-resolved phosphorescence data. We used 300- $\mu$ s-long interexcitation cycles, during which the EOM was in the opened state during the initial $10 \mu \mathrm{s}$ to provide an excitation gate, followed by a $290-\mu$ s-long phosphorescence collection period. We averaged 500 interexcitation cycles during $\sim 150 \mathrm{~ms}$ to collect the decay at each measurement location. In each imaging plane, we selected 30 to 60 locations inside the microvascular segments. Measurement of $\mathrm{pO}_{2}$ at selected locations took 5 to $10 \mathrm{~s}$. The digital acquisition card (National Instruments, PCIe-6537) was used at a $50 \mathrm{MS} / \mathrm{s}$ acquisition rate to collect PMT photon counts after the discriminator (Hamamatsu, C9744). The photon counts were binned into $2-\mu \mathrm{s}$ bins. The first $5 \mu \mathrm{s}$ of the data after the end of the excitation gate were rejected to match the probe calibration conditions and the remaining $285 \mu$ s of phosphorescence decays were fitted with a single exponential function to calculate the decay time. The phosphorescence decay times (lifetimes) were converted to absolute $\mathrm{pO}_{2}$ values using a SternVolmer-like expression obtained in independent calibration experiments, which were performed using solutions of the probe in a physiological buffer (phosphate, $20 \mathrm{mM}$ ) at $36.6^{\circ} \mathrm{C}$. Throughout the range assessed by our calibrations $\left(\mathrm{pO}_{2}=0\right.$ to $\left.160 \mathrm{mmHg}\right)$, the probe displayed nearly ideally linear Stern-Volmer behavior, i.e., the quenching rate constant changed linearly with $\mathrm{pO}_{2}$. In the hyperoxia experiments, we assumed that this linearity is retained at $\mathrm{pO}_{2}$ values above $160 \mathrm{mmHg}$, and thus the hyperoxia values shown in Fig. 5 were obtained by extrapolation of the oxygen titration curve to the higher $\mathrm{pO}_{2}$ region. This extrapolation was justified by the linearity of the Stern-Volmer plot throughout the physiological range (0 to $160 \mathrm{mmHg}$ ) and the fact that the SternVolmer approximation, i.e., the assumption about zero-order of the quenching reaction with respect to quencher $\left(\mathrm{O}_{2}\right)$, should become even more justified physically at higher oxygen concentrations. The objective was translated along the optical $Z$ axis as $\mathrm{pO}_{2}$ measurements were proceeded to different axial planes (typically six to seven per experiment). The measurement results were classified according to three retinal depth ranges. The survey intensity images in each group were merged by maximum intensity projection (MIP), i.e., selecting the maximum intensity value per pixel. The resulting MIP image was contrast enhanced for better visualization and segmentation of the microvascular branches. The resulting MIP images and $\mathrm{pO}_{2}$ measurements were overlaid. Within each vessel segment, $\mathrm{pO}_{2}$ was typically measured in one to five locations (as shown in Fig. 4), then the average $\mathrm{pO}_{2}$ values were computed per vessel segment if more than one point was measured. Finally, the colors (using MATLAB colormap “jet”) were assigned to the entire vessel segments showing average $\mathrm{pO}_{2}$ in $\mathrm{mmHg}$ (Fig. 5).

\subsection{Statistics}

The individual $\mathrm{pO}_{2}$ measurements with standard error higher than $15 \%$ were rejected. Reported $\mathrm{pO}_{2}$ values within major vessels were presented as mean \pm standard deviation (SD), where mean $\mathrm{pO}_{2}$ and its standard deviation were calculated across multiple $\mathrm{pO}_{2}$ measurements within a vascular segment.

\section{Results}

\subsection{Calibration of the Compound Eye-Objective Optical System}

The compound optical system, which consisted of the imaging objective and the intrinsic optical system of the eye, was calibrated in order to quantify the distances within the measured tissue volume in both lateral and axial dimensions. The optical system of the mouse eye, i.e., cornea, aqueous chamber, lens, vitreous chamber, and retina, can be represented by volumes with different refractive indices separated by curved interfaces (Fig. 1, inset).

The dimensions of the mouse eye and hence its optical properties vary with age and strain. We used the previously published data $^{34-36}$ to estimate the optical properties of the eye of the 3-month-old C57BL/6 mice that were used in our experiments (see Table 1) at the excitation wavelength of $950 \mathrm{~nm}$. 
Table 1 Optical parameters used to calculate the depth of the imaging plane within the retina and corresponding lateral magnification. ${ }^{34-36}$

\begin{tabular}{lccccc}
$\begin{array}{l}\text { Effective focal length } \\
f_{\text {eye }}=3.1 \mathrm{~mm}\end{array}$ & Retina & $\begin{array}{l}\text { Vitreous } \\
\text { chamber }\end{array}$ & Lens & $\begin{array}{l}\text { Aqueous } \\
\text { chamber }\end{array}$ & Cornea \\
\hline Thickness/depth & $224 \mu \mathrm{m}$ & $576 \mu \mathrm{m}$ & $2169 \mu \mathrm{m}$ & $295 \mu \mathrm{m}$ & $116 \mu \mathrm{m}$ \\
Refractive index & 1.33 & 1.33 & 1.64 & 1.33 & 1.39 \\
$\begin{array}{l}\text { Radius of curvature } \\
\text { at the interface }\end{array}$ & $\begin{array}{l}\text { Anterior: }-1.52 \mathrm{~mm} ; \\
\text { posterior: }-1.61 \mathrm{~mm}\end{array}$ & $\mathrm{~N} / \mathrm{A}$ & $\begin{array}{c}\text { Anterior: } 1.21 \mathrm{~mm} ; \\
\text { posterior: }-1.08 \mathrm{~mm}\end{array}$ & $\mathrm{~N} / \mathrm{A}$ & $\begin{array}{l}\text { Anterior: } 1.41 \mathrm{~mm} \text {; } \\
\text { posterior: } 1.42 \mathrm{~mm}\end{array}$ \\
\hline
\end{tabular}

We constructed the compound ray transfer matrix of the mouse eye, using the previously published approach, ${ }^{36}$ taking into account the propagation of light through the cornea, lens, aqueous, and vitreous chambers, and the diffraction at the interfaces between them. The whole eye was modeled as a single optical element (i.e., a thick lens). Marginal rays were traced from both directions (cornea-to-retina and retina-to-cornea) in order to calculate front and back focal points, principal planes, and effective focal length $(3.1 \mathrm{~mm})$. We then used the lens equation to estimate the distances [Fig. 2(a)] and magnification [Fig. 2(b)] in our measurements. To determine the lateral dimensions, we focused the excitation beam on the inner-retina surface, translated the animal in $X$ - and $Y$-directions at known distances and recorded fluorescence images of the vasculature (using FITC-dextran as vascular marker) at each position. The shifts between the images in $X$ - and $Y$-dimensions were quantified and scaled with the depth-dependent magnification factor [Fig. 2(b)].

The working distance of the objective lens was quickly depleted as deeper layers of retina were imaged [Fig. 2(a)]. The entire retinal depth $(\sim 220 \mu \mathrm{m})$ was accessible by adjusting the gap between the surfaces of the objective lens and the cornea from 2.7 to $1.6 \mathrm{~mm}$. Upon lowering the focal plane deeper into the retina, the magnification factor induced by the eye optics changed from 2.2 to 2.7. These calibration experiments revealed that our optical system had sufficient working distance and could be used to image through the entire retinal microvascular plexus.

\subsection{Depth-Resolved Imaging of Retinal Microvasculature}

After the optical system was calibrated, we demonstrated the capability of our imaging setup to generate high-resolution
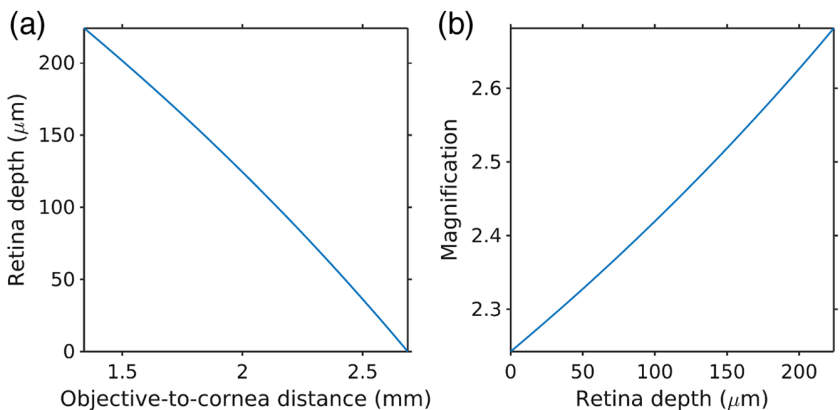

Fig. 2 Calibration of the compound eye-objective optical system. (a) Calibrated retina depth as a function of the distance between the objective lens and the cornea surface. (b) Calibrated lateral magnification introduced by mouse eye optics as a function of imaging plane depth inside the retina. volumetric angiograms by two-photon excited fluorescence of FITC-labeled blood plasma.

Mouse retina is holangiotic, i.e., it does not have an avascular central region, unlike human retina. ${ }^{39}$ Major retinal vessels on the superficial layer are arranged in a radiating star-like pattern consisting of arteries and veins in alternating order [Fig. 3(a)]. The total retinal thickness is $\sim 220 \mu \mathrm{m},{ }^{34}$ and the outer retinal layers $(\sim 100-\mu \mathrm{m}$ thick $)$ are avascular and positioned close to the choroid plexus. ${ }^{15,40}$ The inner retinal layers $(\sim 100-\mu$ m thick $)$ comprise three microvascular layers. ${ }^{41}$ Starting from the retinal surface, these layers are: (1) the superficial layer, which is rich in arterioles and located in the ganglion cell layer; (2) the intermediate layer, located in the inner plexiform layer; and (3) the deep layer, which is rich in venules and located in the outer plexiform layer. $^{40-43}$

Figures 3(b) and 3(c) show images of the retinal microvasculature in two mice. The microvascular structures shown in three panels in Figs. 3(b) and 3(c) represent mostly microvascular segments from the three individual retinal microvascular layers, with little overlap with the vasculature from the adjacent microvascular layers. This confirms that our setup is capable of high-resolution 3-D imaging in the eye and able to distinguish between individual arterioles, venules, and capillaries from different microvascular layers as well as between major arteries and veins.

\subsection{Depth-Resolved Measurement of $\mathrm{pO}_{2}$ in Retinal Microvasculature}

We quantified absolute $\mathrm{pO}_{2}$ in the blood-plasma using the phosphorescence quenching method.

A representative phosphorescence intensity image and the locations of the local microvascular $\mathrm{pO}_{2}$ measurements in the superficial layer are shown in Fig. 4(a). $\mathrm{pO}_{2}$ was measured in a large set of microvascular segments within $438 \times 438 \mu \mathrm{m}^{2}$ field-of-view (FOV), including in arterioles, venules, and capillaries. Representative phosphorescence decays (average photon counts during interexcitation interval of $300 \mu \mathrm{s}$ ) corresponding to a major artery and a vein under normoxia (systemic arterial $\mathrm{pO}_{2}=95.2 \mathrm{mmHg}$ according to blood gas analyzer) are shown in Fig. 4(b). These decays were fitted with single exponentials to derive the phosphorescence lifetimes and subsequently the $\mathrm{pO}_{2}$ values.

Depth-resolved $\mathrm{pO}_{2}$ measurements in the retinal microvasculature, performed in the same mouse during normoxia and hyperoxia, are shown in Fig. 5. We measured $\mathrm{pO}_{2}$ in a major artery, a major vein, a number of arterioles, venules, and capillaries within the superficial, intermediate, and deep retinal microvascular plexus. During normoxia, the mean $\mathrm{pO}_{2}$ values in the major artery and vein were $93 \pm 3 \mathrm{mmHg}$ (mean $\pm \mathrm{SD}$ ) and $63 \pm 2 \mathrm{mmHg}$ (mean $\pm \mathrm{SD})$, respectively. The arterial 
(a)
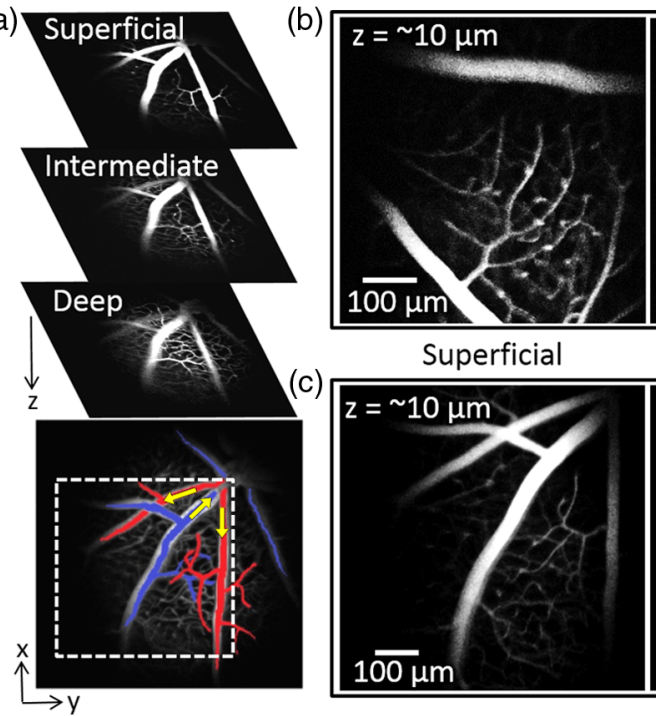

Superficial
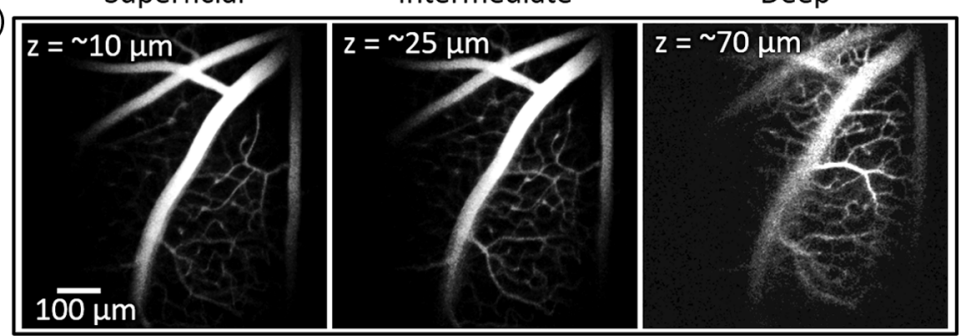

Fig. 3 Two-photon depth-resolved retinal microvasculature imaging. (a) Microvascular structures, as imaged using a low-magnification objective, of the three main microvascular layers in the mouse retina: superficial, intermediate, and the deep. The superficial microvascular layer shows a radiating pattern of major arteries and veins falsely colored in red and blue, respectively. Yellow arrows indicate the direction of the blood flow. ROI is shown by white-dashed square. (b) and (c) Retinal microvasculature layers of two mice, imaged using high-magnification objective. Three imaging depths $(z)$ in (b) and (c) show microvascular structure in three retinal microvascular layers. The imaging depths $(z)$ shown in the graphs represent calculated values based on the calibrations (see the main text and Fig. 2 for details).

(a)

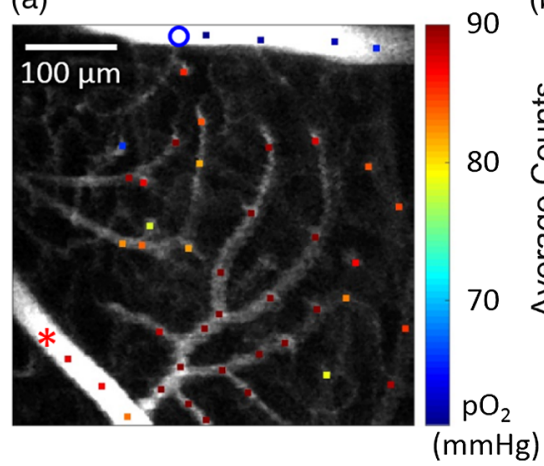

(b)

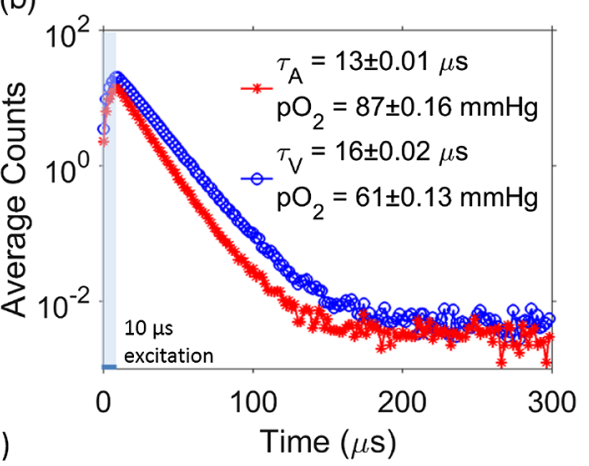

Fig. 4 Two-photon imaging of retinal microvascular $\mathrm{pO}_{2}$. (a) Intravascular $\mathrm{pO}_{2}$ (color coded, color bar in $\mathrm{mmHg}$ ) at various locations within retinal microvasculature. $\mathrm{pO}_{2}$ measurement locations are marked by colored dots on the gray-scale survey intensity image. (b) Examples of phosphorescence decays measured within the artery (red) and vein (blue). The measurement locations corresponding to the decays shown were marked on image (a) with a red star and a blue circle, respectively. $10-\mu \mathrm{s}$-long laser excitation gate was marked with the shaded area at the beginning of the interexcitation interval. Fitted phosphorescence lifetimes in arteriole and venule $\left(\tau_{A}\right.$ and $\tau_{V}$, respectively) and their corresponding $\mathrm{pO}_{2}$ values are provided inside the panel. Values are presented as mean \pm standard error of mean (SEM).

value was consistent with the systemic arterial $\mathrm{pO}_{2}=$ $112 \mathrm{mmHg}$, measured using a blood gas analyzer. Longitudinal $\mathrm{pO}_{2}$ gradients, starting from high $\mathrm{pO}_{2}$ in the major artery at the superficial retinal layer, could be visualized along the precapillary arterioles as approaching lower $\mathrm{pO}_{2}$ values in the capillaries close to the postcapillary venules, and finally to the major vein.

We subsequently perturbed the microvascular oxygenation in the retina by inducing hyperoxia, whereby the systemic arteriolar $\mathrm{pO}_{2}$ was increased to $334 \mathrm{mmHg}$. Similar to the measurements under normoxia, the longitudinal gradients of intravascular $\mathrm{pO}_{2}$ could be visualized between the major artery $(327 \pm 2 \mathrm{mmHg}$, mean $\pm \mathrm{SD})$ and vein $(224 \pm 4 \mathrm{mmHg}$, mean $\pm \mathrm{SD}$ ) in the superficial retinal layer, i.e., the small vascular segments close to the major artery were more oxygenated than those close to the major vein.

The $\mathrm{pO}_{2}$ values in the major artery and vein, as well as in the combined superficial, intermediate, and deep microvascular plexus (microvascular bed) under normoxia and hyperoxia are shown in Fig. 6. During normoxia, the median $\mathrm{pO}_{2}$ values in the major artery, vein, and in the microvascular bed were 93, 62, and $87 \mathrm{mmHg}$, respectively, with the microvascular bed $\mathrm{pO}_{2}$ distribution being highly heterogeneous, spanning the range of $\mathrm{pO}_{2}$ values from arteriolar to venular $\mathrm{pO}_{2}$. 

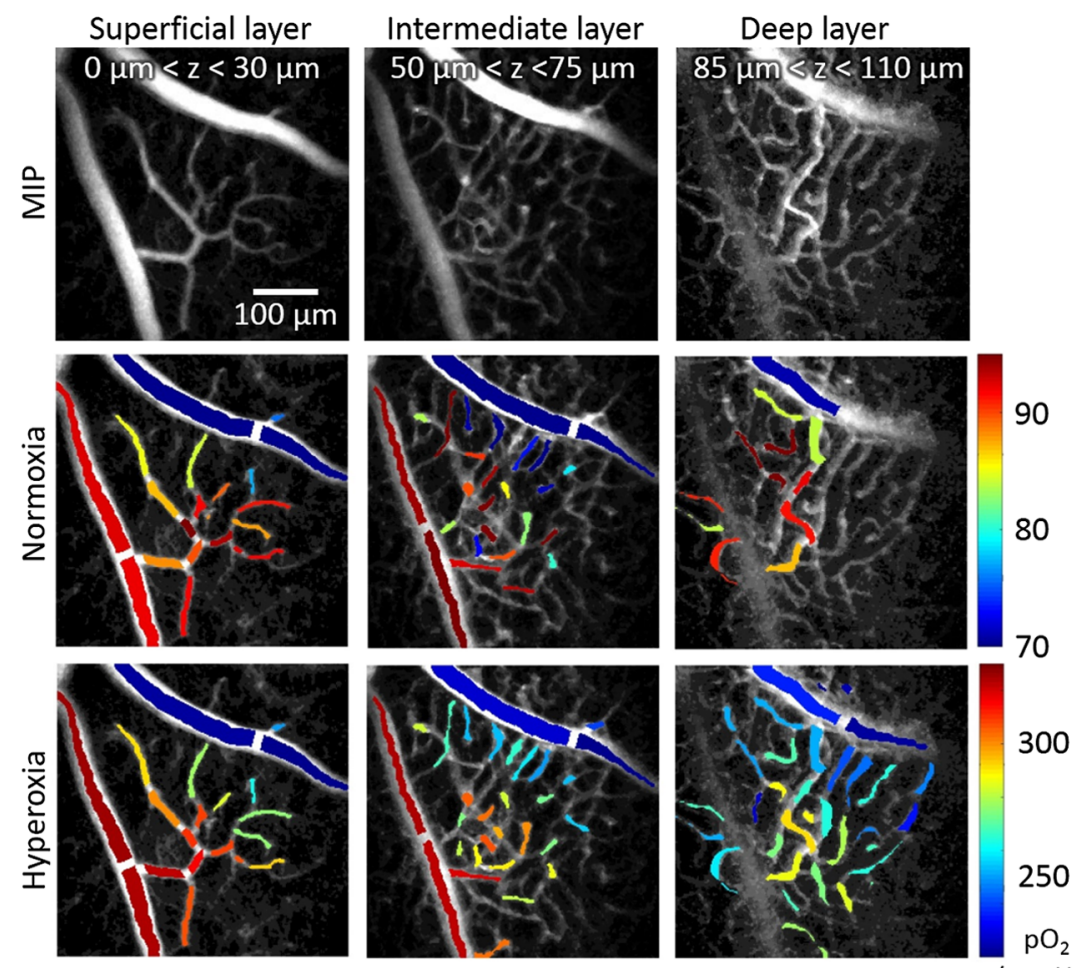

$(\mathrm{mmHg})$

Fig. $5 \mathrm{pO}_{2}$ distribution in the retinal microvasculature during normoxia and hyperoxia. Survey images (top) and $\mathrm{pO}_{2}$ measurements superimposed on these images during normoxia (middle row) and hyperoxia (bottom row). Columns represent different microvascular layers: superficial (first column), intermediate (middle column), and deep layer (third column). Vascular segment colors in second and third rows represent average of all $\mathrm{pO}_{2}$ point measurements within the vascular segment (colorbar in $\mathrm{mmHg}$ ). Colorcoded masks were overlaid on the gray-scaled MIP survey scan images. Both the imaging depth ranges $(z)$ and the dimensions of the FOV were estimated using the calibration curves presented in Fig. 2. Note that the $\mathrm{pO}_{2}$ ranges during normoxia and hyperoxia are far apart, so we used separate $\mathrm{pO}_{2}$ color scales to aid visualization of $\mathrm{pO}_{2}$ distributions in the retinal microvasculature at these two conditions.
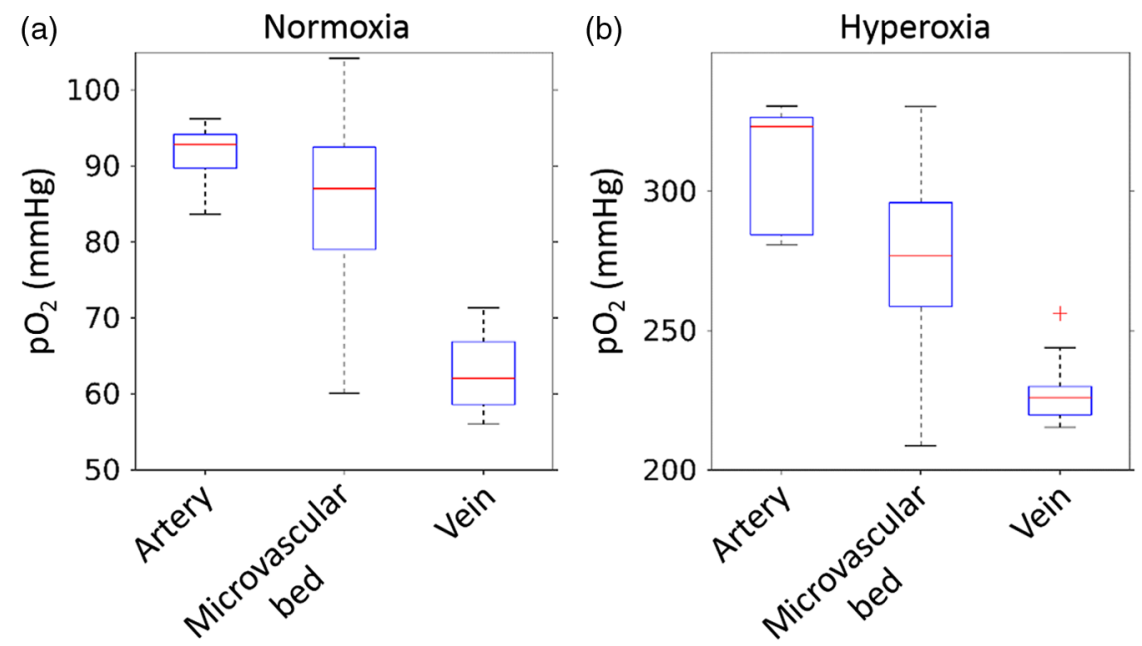

Fig. $6 \mathrm{pO}_{2}$ distribution across different retinal compartments: artery, vein, and microvascular bed in between, under (a) normoxic and (b) hyperoxic conditions (same data as in Fig. 5). The top and bottom edges of the boxes (shown in blue) mark the 75th and 25th percentiles; the error bars show the extreme ends of the data spread (red + symbols), excluding outliers, and the red-center lines mark the medians.

Using the Hill equation with coefficients characteristic of C57BL/6 mice $\left(h=2.59\right.$ and $\left.P_{50}=40.2\right),{ }^{44}$ we calculated the hemoglobin oxygen saturation $\left(\mathrm{sO}_{2}\right)$ in the major artery and vein during normoxia. The corresponding values were $90 \%$ and $75 \%$, respectively. These results suggest that the oxygen extraction fraction was $16 \%$. During hyperoxia, the median $\mathrm{pO}_{2}$ in the artery, vein, and the microvascular bed were 323,226 , and $277 \mathrm{mmHg}$, respectively, with the microvascular bed $\mathrm{pO}_{2}$ distribution, as in the normoxic case, being highly heterogeneous and spanning the range of $\mathrm{pO}_{2}$ values from arteriolar to venular $\mathrm{pO}_{2}$. 


\section{Discussion and Conclusion}

Here, we performed in vivo measurements of absolute $\mathrm{pO}_{2}$ in major retinal vessels, and, for the first time, in arterioles, venules, and capillaries within three depth-resolved microvascular plexus, by 2PLM, using a potent two-photon phosphorescent oxygen probe. Oxygen measurements by phosphorescence quenching are based on the probe's triplet decay time (phosphorescence lifetime), and as such they are unaffected neither by variations in the local probe concentration nor by optical properties of the medium (tissue) ${ }^{45}$ Compared to an earlier two-photon oxygen probe PtP-C $343 .{ }^{31} \mathrm{Pt}$ tetraphthalimidoporphyrin ${ }^{32}$ has a significantly red-shifted emission spectrum, centered at $757 \mathrm{~nm}$ and $\sim 50$ - to 100-fold higher two-photon brightness. As a result, it allows much deeper and faster measurements of $\mathrm{pO}_{2}$ in vivo. In this pilot study, we conservatively averaged 500 decays at each measurement location (e.g., $150 \mathrm{~ms}$ per point measurement); however, in the future it should be possible to shorten the acquisition time by reducing the number of cycles per location, while still maintaining adequate signal-to-noise ratio for accurate $\mathrm{pO}_{2}$ determination.

In vivo $2 \mathrm{PM}$ imaging of the retinal tissue with high spatial resolution presents specific challenges to the optical setup design due to the intrinsic optical properties of the eye. When imaging the retina in an intact eye through the pupil, one should consider that: (1) the imaging plane is located deeper than expected from the physical distance between the surface of the cornea and the retina ( $\sim 3 \mathrm{~mm}$ in mice) due to the refractive properties of the eye itself; (2) when using objectives with large numerical apertures (NA), the diameter of the pupil may limit the aperture size of the imaging system and compromise resolution; (3) the compound (objective plus eye) imaging system is prone to strong aberrations limiting the useful FOV.

The crystalline lens of the eye works together with the imaging objective to focus the excitation beam on a more anterior plane within the eye than expected from the objective working distance. Therefore, traditional 2PM objectives with working distances of 2 to $3 \mathrm{~mm}$ are not suitable for retinal imaging. This challenge has been addressed in the past by using adaptive optics combined with an animal-specific contact lenses laid over the cornea, ${ }^{46}$ or using a more flexible periscope design. ${ }^{47}$ Bar-Noam et al. ${ }^{48}$ placed a convex electrically tunable lens and a concave offset lens before a water-immersion $10 \times$ microscope objective (WD $1.8 \mathrm{~mm}$ or $3.5 \mathrm{~mm}$ ) in order to extend their imaging depth to cover the entire retinal range. However, using either tunable lens or adaptive optics substantially increases the complexity of the imaging setup. Being able to perform 2PM imaging of the retina with a standard 2PM system should be advantageous. We used a commercially available 2PM objective with large working distance, which was coupled to the surface of the cornea to image retinal microvascular layers. Using this objective, we were able to image throughout the entire retinal depth although the working distance was effectively shortened by the optics of the eye. It should be noted that the eye's iris limits the aperture and hence the FOV and the effective NA of the overall imaging system. Therefore, in spite of the fact that imaging was performed through a dilated pupil ( $\sim 2 \mathrm{~mm}$ in diameter), full NA of the objective was not utilized.

During in vivo imaging of retina, contributions of the eye optics to the imaging cannot be avoided. Therefore, the compound optical system needs to be calibrated to ensure correct estimation of imaging dimensions and depths. This was achieved in the past by: (1) using histological images of the retina as a reference to scale the corresponding in vivo imaged en face planes; ${ }^{46,49}$ (2) assuming the focal length of the eye lens and scaling the magnification factor of the combined optical system accordingly; ${ }^{47}$ and (3) employing ray tracing or software, such as Zemax (Zemax, LLC) in combination with the assumed optical properties of the eye. ${ }^{48}$ In our approach, at the time of coupling, the distance between the objective and the cornea was not known. We used the star-shaped pattern of the major vessels as a guide to set the focus on the retina surface. We then assigned the depth at which focusing occurred as a reference and calculated the axial distances and magnifications relative to this depth using a ray matrix model of the optics of the mouse eye. Furthermore, we calibrated the lateral distances by introducing known displacements in the lateral plane (X - Y) while the beam was focused on the retinal surface, and subsequently scaled them using the previously calculated depth-dependent magnification factor.

Our 2PM setup has an axial resolution of $\sim 5 \mu \mathrm{m}$ and lateral resolution of $\sim 2 \mu \mathrm{m}^{33}$ for $\mathrm{pO}_{2}$ measurements. Despite additional challenges (optical aberrations, etc.) induced by imaging through the eye, the spatial resolution of our imaging system was sufficient to visualize different retinal microvascular layers. The relatively sparse configuration of the capillaries within the retinal microvascular bed also helped to avoid potential artifacts due to the overlaps between the sampled volumes when the beam was focused at neighboring vessels.

We validated our system's capability to assess retinal oxygenation by measuring intravascular $\mathrm{pO}_{2}$ during normoxia and hyperoxia. Systemic arterial $\mathrm{pO}_{2}$ and retinal arterial $\mathrm{pO}_{2}$ measurements were in good agreement under normoxia (112 versus $93 \mathrm{mmHg}$ ) and under hyperoxia (334 versus $327 \mathrm{mmHg}$ ). It could be expected that the systemic arterial $\mathrm{pO}_{2}$ would be somewhat higher than the $\mathrm{pO}_{2}$ in retinal arterioles due to the oxygen extraction from the arterioles. Previous results from our group and others revealed longitudinal $\mathrm{pO}_{2}$ gradients in arterioles $^{50-52}$ as well as $\mathrm{pO}_{2}$ gradients in periarteriolar tissue. ${ }^{51,53-55}$ Under both normoxia and hyperoxia, we were able to visualize longitudinal $\mathrm{pO}_{2}$ gradients throughout the microvascular bed. However, in the case of hyperoxia, the gradients could be in part due to the change in the concentration of oxygen that is dissolved in the blood plasma, without significant change in $\mathrm{sO}_{2}$, since blood hemoglobin would be almost fully saturated with oxygen under hyperoxic conditions. Motion artifacts due to heavy breathing as well as reduced tissue transparency after prolonged anesthesia may hamper measurements in every capillary as displayed in the deep retina layer (Fig. 5, normoxia).

Several studies have previously reported $\mathrm{pO}_{2}$ measurements in major retinal arteries and veins of mice using phosphorescence lifetime imaging of different single-photon excited oxyphors. In one of our earlier studies, ${ }^{25}$ we used a phosphorescent probe based on Pd tetracarboxyphenylporphyrin (Frontier Scientific, Logan, Utah, single-photon excitation at $532 \mathrm{~nm}$ ) to measure intravascular $\mathrm{pO}_{2}$ in 3-month-old C57BLI6J mice under ketamine/xylazine anesthesia, and reported arterial $\mathrm{pO}_{2}$ of $36 \pm 7 \mathrm{mmHg}$ and venular $\mathrm{pO}_{2}$ of $22 \pm 5 \mathrm{mmHg}$ $($ mean $\pm \mathrm{SD})$. Wilson et al. ${ }^{21}$ measured retinal intravascular $\mathrm{pO}_{2}$ using Oxyphor $\mathrm{G} 2^{56,57}$ in mice under ketamine/xylazine anesthesia with single-photon excitation at $450 \mathrm{~nm}$. The reported $\mathrm{pO}_{2}$ values in arterioles, venules, and capillary regions were 60 to 80,30 to 45 , and 50 to $100 \mathrm{mmHg}$, respectively. ${ }^{21}$ 
Prior to that, Shonat and $\mathrm{Kight}^{20}$ performed imaging using single-photon excitation at $524 \mathrm{~nm}$ and reported $\mathrm{pO}_{2}$ values of $59 \pm 5 \mathrm{mmHg}$ in arterioles, $45 \pm 5 \mathrm{mmHg}$ in venules, and $80 \pm 10 \mathrm{mmHg}$ (mean $\pm \mathrm{SD}$ ) in capillary bed halothaneanesthetized C57BLI6 mice. ${ }^{20}$ The $\mathrm{pO}_{2}$ values reported here are generally higher than those found in the previous studies. ${ }^{20,21,25}$ It is possible that the choice of animal strain, age, and, importantly, type and duration of anesthesia, contributed to this difference. It is also important to note that the measurement methods used in all of the previous studies had limited depth resolution, and thus the reported values may have been effectively convolutions of the $\mathrm{pO}_{2}$ values at different depths throughout the sampled volumes of the retina.

Although $\mathrm{pO}_{2}$ in major vessels close to the retinal surface has been quantified previously, our work is the first demonstration of $\mathrm{pO}_{2}$ measurements in retinal capillaries at different depths with single-capillary resolution. This advance is important because the main oxygen transport to tissue occurs at the capillary sites. Moreover, the early-phase clinical indications of retinal pathologies are associated with capillaries, and our method may open up a new avenue to study baseline and transient $\mathrm{pO}_{2}$ in capillaries in health and various mouse models of retinal diseases.

In this work, extrapolation of the probe calibration to the hyperoxia regime may have resulted in a slight, most likely constant overestimation of the $\mathrm{pO}_{2}$ values at $\mathrm{pO}_{2} \mathrm{~s}$ above $160 \mathrm{mmHg}$. However, this discrepancy, even if present, should not change the key result of our proof-of-principle hyperoxia experiments, i.e., the ability of our setup to detect perturbations in retinal oxygenation as well as longitudinal gradients from arterioles to capillaries to venules. In the future, with sufficient interest in quantification of biological hyperoxia, we may perform detailed calibrations of our probes in the upper $\mathrm{pO}_{2}$ range.

Mouse eye has no apparent sensitivity in the spectral range of our excitation and emission $(950 \mathrm{~nm} / 757 \mathrm{~nm}) .{ }^{58}$ Thus, we presume that our measurements did not cause direct visual stimulation. On the other hand, a few endogenous chromophores in the retina (e.g., melanin and lipofuscin) have measurable twophoton absorption cross-sections near $950 \mathrm{~nm}$ as well as onephoton absorption spectra near $757 \mathrm{~nm} .{ }^{59}$ Hence, it is possible that some indirect visual stimulation during the experiment affected the retinal metabolism. However, melanin and lipofuscin accumulations are mostly confined to the retinal pigment epithelial and subretinal microglia cells ${ }^{60}$ that are positioned significantly below the focal planes imaged in our experiments. In addition, lipofuscin accumulation in retina is insignificant in animals younger than 12 months. ${ }^{60}$ It is important to note that our $\mathrm{pO}_{2}$ measurements are not compromised by autofluorescence, since they rely on the triplet decay that is orders of magnitude longer than the time scale of any potential tissue autofluorescence.

Our method can be combined with other imaging techniques that can be used, e.g., for measurements of microvascular blood flow, as previously demonstrated in brain..$^{33,61-67}$ Furthermore, oxygen-sensitive phosphorescent probes can be delivered to the retinal extravascular space. ${ }^{29,30}$ Therefore, we anticipate that 2PLM can be used in the future to perform depth-resolved extravascular retinal $\mathrm{pO}_{2}$ as well, similarly to how it was done previously in the rodent brain cortex. ${ }^{53,33}$ Overall, we anticipate that our method for measuring $\mathrm{pO}_{2}$ in retinal microvasculature will stimulate new studies of the pathophysiology of the eye and lead to better understanding of the mechanisms underlying various retinal disorders.

\section{Disclosures}

The authors declare no conflicts of interest.

\section{Acknowledgments}

This work was supported by the National Institute of Health (NIH) Grant Nos. EY017918, NS092986, EB018464, NS091230, NS055104, HL133362, MH111359, AG042026, and AA027097.

\section{References}

1. M. W. Country, "Retinal metabolism: a comparative look at energetics in the retina," Brain Res. 1672(Suppl. C), 50-57 (2017).

2. T. Y. Wong et al., "Diabetic retinopathy," Nat. Rev. Dis. Primer 2, 16012 (2016).

3. C. A. Shah, "Diabetic retinopathy: a comprehensive review," Indian J. Med. Sci. 62(12), 500-519 (2008).

4. "Statistics and DatalNational Eye Institute," https://nei.nih.gov/eyedata (19 April 2018)

5. R. S. Eshaq, W. S. Wright, and N. R. Harris, "Oxygen delivery, consumption, and conversion to reactive oxygen species in experimental models of diabetic retinopathy," Redox Biol. 2, 661-666 (2014).

6. D.-Y. Yu and S. J. Cringle, "Oxygen distribution in the mouse retina," Invest. Ophthalmol. Vis. Sci. 47(3), 1109-1112 (2006).

7. J. C. M. Lau and R. A. Linsenmeier, "Oxygen consumption and distribution in the Long-Evans rat retina," Exp. Eye Res. 102, 50-58 (2012).

8. S. J. Cringle et al., "Intraretinal oxygen consumption in the rat in vivo," Invest. Ophthalmol. Vis. Sci. 43(6), 1922-1927 (2002).

9. T. E. Kornfield and E. A. Newman, "Regulation of blood flow in the retinal trilaminar vascular network," J. Neurosci. 34(34), 11504-11513 (2014).

10. J. C. M. Lau and R. A. Linsenmeier, "Increased intraretinal $\mathrm{pO}_{2}$ in short-term diabetic rats," Diabetes 63(12), 4338-4342 (2014).

11. T. S. Hwang et al., "Automated quantification of nonperfusion areas in 3 vascular plexuses with optical coherence tomography angiography in eyes of patients with diabetes," JAMA Ophthalmol. 136(8), 929-936 (2018).

12. A. C. Onishi et al., "Importance of considering the middle capillary plexus on OCT angiography in diabetic retinopathy," Invest. Ophthalmol. Vis. Sci. 59(5), 2167-2176 (2018).

13. S. H. Hardarson et al., "Automatic retinal oximetry," Invest. Ophthalmol. Vis. Sci. 47(11), 5011-5016 (2006).

14. A. Hariri et al., "In vivo photoacoustic imaging of chorioretinal oxygen gradients," J. Biomed. Opt. 23(3), 036005 (2018).

15. J. Yi et al., "Visible light optical coherence tomography measures retinal oxygen metabolic response to systemic oxygenation," Light Sci. Appl. 4(9), e334 (2015).

16. S. P. Chong et al., "Quantitative microvascular hemoglobin mapping using visible light spectroscopic optical coherence tomography," Biomed. Opt. Express 6(4), 1429-1450 (2015).

17. S. P. Chong et al., "Ultrahigh resolution retinal imaging by visible light OCT with longitudinal achromatization," Biomed. Opt. Express 9(4), 1477-1491 (2018).

18. J. M. Vanderkooi et al., "An optical method for measurement of dioxygen concentration based upon quenching of phosphorescence," J. Biol. Chem. 262(12), 5476-5482 (1987).

19. R. D. Shonat et al., "Oxygen distribution in the retinal and choroidal vessels of the cat as measured by a new phosphorescence imaging method," Appl. Opt. 31(19), 3711-3718 (1992).

20. R. D. Shonat and A. C. Kight, "Oxygen tension imaging in the mouse retina," Ann. Biomed. Eng. 31(9), 1084-1096 (2003).

21. D. F. Wilson et al., "Oxygen distribution and vascular injury in the mouse eye measured by phosphorescence-lifetime imaging," Appl. Opt. 44(25), 5239-5248 (2005).

22. M. Shahidi et al., "A method for chorioretinal oxygen tension measurement," Curr. Eye Res. 31(4), 357-366 (2006).

23. J. Wanek et al., "Inner retinal metabolic rate of oxygen by oxygen tension and blood flow imaging in rat," Biomed. Opt. Express 2(9), 2562-2568 (2011). 
24. J. Wanek et al., "Inner retinal oxygen delivery and metabolism under normoxia and hypoxia in rat," Invest. Ophthalmol. Vis. Sci. 54(7), 5012-5019 (2013).

25. N. P. Blair et al., "Inner retinal oxygen delivery, metabolism, and extraction fraction in ins2akita diabetic mice," Invest. Ophthalmol. Vis. Sci. 57(14), 5903-5909 (2016).

26. H. Akiyama et al., "Ir(III) complex-based oxygen imaging of living cells and ocular fundus with a gated ICCD camera," Photochem. Photobiol. Sci. 17(6), 846-853 (2018).

27. M. Shahidi et al., "Three-Dimensional mapping of chorioretinal vascular oxygen tension in the rat," Invest. Ophthalmol. Vis. Sci. 50(2), 820-825 (2009).

28. P. Teng et al., "Oxygen tension and gradient measurements in the retinal microvasculature of rats," Graefes Arch. Clin. Exp. Ophthalmol. 250(3), 361-367 (2012).

29. M. Shahidi et al., "Retinal tissue oxygen tension imaging in the rat," Invest. Ophthalmol. Vis. Sci. 51(9), 4766-4770 (2010).

30. A. E. Felder et al., "A method for volumetric retinal tissue oxygen tension imaging," Curr. Eye Res. 43(1), 122-127 (2018).

31. O. S. Finikova et al., "Oxygen microscopy by two-photon-excited phosphorescence," Chem. Phys. Chem. 9(12), 1673-1679 (2008).

32. T. V. Esipova et al., "Stabilizing g-states in centrosymmetric tetrapyrroles: two-photon-absorbing porphyrins with bright phosphorescence," J. Phys. Chem. A 121(33), 6243-6255 (2017).

33. M. A. Yaseen et al., "Multimodal optical imaging system for in vivo investigation of cerebral oxygen delivery and energy metabolism," Biomed. Opt. Express 6(12), 4994-5007 (2015).

34. C. Schmucker and F. Schaeffel, "A paraxial schematic eye model for the growing C57BL/6 mouse," Vision Res. 44(16), 1857-1867 (2004).

35. H. Zhang et al., "The measurement of corneal thickness from center to limbus in vivo in C57BL/6 and BALB/c mice using two-photon imaging," Exp. Eye Res. 115, 255-262 (2013).

36. G. Bawa et al., "Variational analysis of the mouse and rat eye optical parameters," Biomed. Opt. Express 4(11), 2585-2595 (2013).

37. F. C. Delori, R. H. Webb, and D. H. Sliney, "Maximum permissible exposures for ocular safety (ANSI 2000), with emphasis on ophthalmic devices," J. Opt. Soc. Am. A 24(5), 1250-1265 (2007).

38. A. Y. Lebedev et al., "Dendritic phosphorescent probes for oxygen imaging in biological systems," ACS Appl. Mater. Interfaces 1(6), 1292-1304 (2009).

39. L. Lossi et al., "Anatomical features for an adequate choice of experimental animal model in biomedicine: II. Small laboratory rodents, rabbit, and pig," Ann. Anat. 204, 11-28 (2016).

40. R. A. Cuthbertson and T. E. Mandel, "Anatomy of the mouse retina. Endothelial cell-pericyte ratio and capillary distribution," Invest. Ophthalmol. Vis. Sci. 27(11), 1659-1664 (1986).

41. D. Ramos et al., "The use of confocal laser microscopy to analyze mouse retinal blood vessels" (2013).

42. M. Paques et al., "Structural and hemodynamic analysis of the mouse retinal microcirculation," Invest. Ophthalmol. Vis. Sci. 44(11), 49604967 (2003).

43. A. B. Simmons and P. G. Fuerst, "Analysis of retinal vascular plexuses and interplexus connections," Methods Mol. Biol. 1753, 317-330 (2018).

44. K. Uchida, M. P. Reilly, and T. Asakura, "Molecular stability and function of mouse hemoglobins," Zool. Sci. 15(5), 703-706 (1998).

45. S. A. Vinogradov and D. F. Wilson, "Porphyrin dendrimers as biological oxygen sensors," in Designing Dendrimers, S. Campagna, P. Ceroni, and F. Puntoriero, Eds., pp. 463-504, John Wiley \& Sons, Hoboken, New Jersey (2011).

46. G. Palczewska et al., "Noninvasive two-photon microscopy imaging of mouse retina and retinal pigment epithelium through the pupil of the eye," Nat. Med. 20(7), 785-789 (2014).

47. P. Stremplewski et al., "Periscope for noninvasive two-photon imaging of murine retina in vivo," Biomed. Opt. Express 6(9), 3352-3361 (2015).
48. A. S. Bar-Noam, N. Farah, and S. Shoham, "Correction-free remotely scanned two-photon in vivo mouse retinal imaging," Light Sci. Appl. 5(1), e16007 (2016).

49. Y. Geng et al., "Adaptive optics retinal imaging in the living mouse eye," Biomed. Opt. Express 3(4), 715-734 (2012).

50. S. Sakadžić et al., "Large arteriolar component of oxygen delivery implies safe margin of oxygen supply to cerebral tissue," Nat. Commun. 5, 5734 (2014).

51. M. Moeini et al., "Compromised microvascular oxygen delivery increases brain tissue vulnerability with age," Sci. Rep. 8(1), 8219 (2018).

52. E. P. Vovenko and A. E. Chuikin, "Oxygen tension in rat cerebral cortex microvessels in acute anemia," Neurosci. Behav. Physiol. 38(5), 493-500 (2008).

53. A. Devor et al., "'Overshoot' of $\mathrm{O}_{2}$ is required to maintain baseline tissue oxygenation at locations distal to blood vessels," J. Neurosci. 31(38), 13676-13681 (2011).

54. E. Vovenko, "Distribution of oxygen tension on the surface of arterioles, capillaries and venules of brain cortex and in tissue in normoxia: an experimental study on rats," Pflugers Arch. 437(4), 617-623 (1999).

55. S. Sakadžić et al., "Two-photon microscopy measurement of cerebral metabolic rate of oxygen using periarteriolar oxygen concentration gradients," Neurophotonics 3(4), 045005 (2016).

56. I. B. Rietveld, E. Kim, and S. A. Vinogradov, "Dendrimers with tetrabenzoporphyrin cores: near infrared phosphors for in vivo oxygen imaging," Tetrahedron 59(22), 3821-3831 (2003).

57. I. Dunphy, S. A. Vinogradov, and D. F. Wilson, "Oxyphor R2 and G2: phosphors for measuring oxygen by oxygen-dependent quenching of phosphorescence," Anal. Biochem. 310(2), 191-198 (2002).

58. S. N. Peirson et al., "Light and the laboratory mouse," J. Neurosci. Methods 300, 26-36 (2018).

59. C. Dysli et al., "Fluorescence lifetime imaging ophthalmoscopy," Prog. Retinal Eye Res. 60(Suppl. C), 120-143 (2017).

60. H. Xu et al., "Age-dependent accumulation of lipofuscin in perivascular and subretinal microglia in experimental mice," Aging Cell 7(1), 58-68 (2008).

61. J. Lecoq et al., "Simultaneous two-photon imaging of oxygen and blood flow in deep cerebral vessels," Nat. Med. 17(7), 893-898 (2011).

62. M. A. Yaseen et al., "Microvascular oxygen tension and flow measurements in rodent cerebral cortex during baseline conditions and functional activation," J. Cereb. Blood Flow Metab. 31(4), 1051-1063 (2011).

63. V. J. Srinivasan et al., "Depth-resolved microscopy of cortical hemodynamics with optical coherence tomography," Opt. Lett. 34(20), 3086-3088 (2009).

64. B. Jeong et al., "Combined two-photon microscopy and optical coherence tomography using individually optimized sources," Opt. Express 19(14), 13089-13096 (2011).

65. B. Kim et al., "Combined two-photon microscopy and angiographic optical coherence tomography," J. Biomed. Opt. 18(8), 80502 (2013).

66. M. B. Bouchard et al., "Ultra-fast multispectral optical imaging of cortical oxygenation, blood flow, and intracellular calcium dynamics," Opt. Express 17(18), 15670-15678 (2009).

67. A. K. Dunn et al., "Simultaneous imaging of total cerebral hemoglobin concentration, oxygenation, and blood flow during functional activation," Opt. Lett. 28(1), 28-30 (2003).

Ikbal Şencan is a research fellow in Optics@ Martinos at MGH and Harvard Medical School. She completed her BSc degree in electronics engineering at Fatih University as valedictorian. She received her MSc and PhD degrees in electrical engineering from UCLA. She worked at Yale School of Medicine as a postdoctoral associate for two years. She coauthored three book chapters, two patents, 19 peer-reviewed journal articles, $>50$ conference presentations, and has an $h$-index of 15 with $>1550$ citations.

Biographies for the authors are not available. 